\title{
Computed tomography: the investigation of choice for aortic dissection?
}

\author{
H SINGH,* E FITZGERALD, M S T RUTTLEY \\ From the Departments of *Cardiology and Radiology, University of Wales College of Medicine, Heath Park, \\ Cardiff
}

SUMmaRY Computed tomography has become established as complementary to aortography in the investigation of patients with suspected aortic dissection. Two cases of dissecting aneurysm are reported in which extensive aortography failed to show evidence of dissection. In both cases dissection was demonstrated by computed tomography. The diagnosis was confirmed in one case at operation and in the other case by follow up.

It is suggested that computed tomography is the diagnostic method of first choice in aortic dissection.

Effective management of acute dissection of the aorta is based on accurate and prompt diagnosis. Aortography has been the definitive method of diagnosis but computed tomography has recently become established as a complementary, and reliable, diagnostic method. The findings of computed tomography in acute dissection ${ }^{1}$ parallel those of aortography. The diagnosis is confirmed by tomography if contrast material fills two or more channels in the aorta and if an intimal flap is identified, or (when there is no free flow of contrast material through the false channel) if intimal calcification is displaced inward from the aortic wall. Associated but non-diagnostic findings are thombus in the aorta; mediastinal, pericardial, or pleural haematomas; and delayed filling and emptying of parts of the aortic lumen as shown by dynamic scanning after bolus injection of contrast material.

Computed tomography avoids the vascular catheterisation needed for aortography but is limited by low spatial resolution so that aortic branches cannot be assessed reliably. Artefacts can complicate the diagnosis of dissection by computed tomography, ${ }^{1}$ and high quality tomograms may occasionally fail to demonstrate a dissection when one is present. ${ }^{2}$ Nevertheless, several studies assessing computed tomography and aortography in the diagnosis of dissection have shown that the two investigations give comparable results. ${ }^{3-5} \mathrm{We}$ describe two cases in

Requests for reprints to Dr H Singh, University of Wales College of Medicine, Heath Park, Cardiff CF4 4XN.

Accepted for publication 14 April 1986 which computed tomography was better than aortography in the diagnosis of dissection.

\section{Patients and methods}

\section{CASE 1}

A 65 year old man presented with sudden, severe, sharp interscapular pain that had come on while he was driving. For 10 years he had had hypertension, which was treated with acebutolol and bendrofluazide. On admission he was still in pain and hypertensive $(190 / 110 \mathrm{~mm} \mathrm{Hg})$, all the peripheral pulses were normal, there were no murmurs, and he was not in cardiac failure. The electrocardiogram was normal. A portable (anteroposterior) chest $x$ ray suggested a wide superior mediastinum. A clinical diagnosis of acute aortic dissection was made.

At cardiac catheterisation the pressure in the ascending aorta was $160 / 100 \mathrm{~mm} \mathrm{Hg}$ and the left ventricular pressure was $160 / 10 \mathrm{~mm} \mathrm{Hg}$. The aortic root and arch were examined by simultaneous biplane (right and left anterior oblique) cineaortography at 50 frames $/ \mathrm{s}$ on $35 \mathrm{~mm}$ film. Hexabrix $320(60 \mathrm{ml})$ was injected at $30 \mathrm{ml} / \mathrm{s}$ for each aortogram and the output phosphors of Siemens $23 \mathrm{~cm}$ intensifiers were photographed with Arritechno cameras. A third single plane (posteroanterior), 25 frames/s, cineaortogram of the descending thoracic and abdominal aorta was obtained by an otherwise similar technique. Routine cine films of left ventriculography and coronary arteriography completed the procedure and the angiograms were sub- 


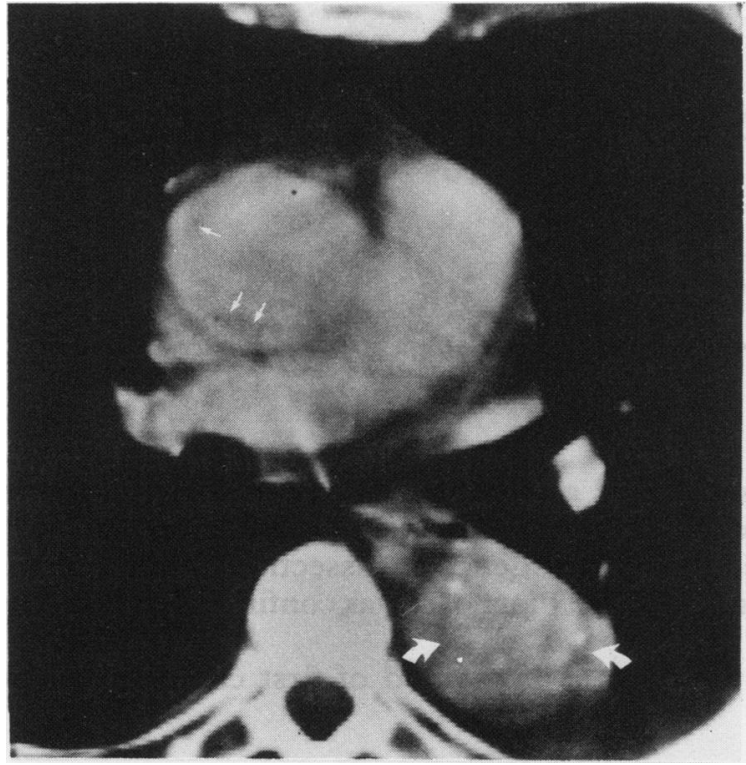

Fig 1 Computed tomography through mid-thorax of case 1 showing an intimal flap in ascending (straight arrows) and descending (curved arrows) aorta. Areas of calcification are seen within the lumen of the descending aorta. There are bilateral pleural effusions.

sequently displayed by a Cipro 35 projector. There was no aortic regurgitation and no sign of aortic dissection, though the thoracic aorta was slightly dilated throughout its course. The ventriculogram was normal and the coronary arteries showed only trivial irregularities.

A posteroanterior chest radiograph taken three days later showed an enlarged heart, wide superior mediastinum, aortic dilatation, and small bilateral pleural effusions. Serum bilirubin concentration rose to $63 \mu \mathrm{mol} / 1$ (normal range $<17 \mu \mathrm{mol} / 1$ ). Thus despite the findings on aortography there was still a strong clinical suspicion of dissection. Eleven days after admission, computed tomography of the thorax and abdomen was performed by means of a Phillips Tomoscan 350 with a dynamic scanning program capable of obtaining 30 images within $5 \mathrm{~min}$. Contrast enhancement was performed with $150 \mathrm{ml}$ of Omnipaque 300 given by rapid peripheral venous injection. A dissecting aneurysm, extending from just above the aortic valve to the level of the aortic hiatus, was shown (fig 1). Pleural effusions were noted bilaterally.

Because the dissection shown by tomography affected the ascending and descending thoracic aorta, operation was considered to be necessary and he was transferred to another specialist centre for this. Aortography was repeated there (fig 2) and again showed no evidence of dissection; because of this and the patient's stable clinical condition, operation was not performed and the patient was discharged for outpatient follow up.

Six months later, computed tomography of the thorax was repeated. The pleural effusions had resolved. The diameter of the aorta in the lower thoracic region had increased to $7 \mathrm{~cm}$, calcified thrombus was seen within the aorta (fig 3 ), and the crescent
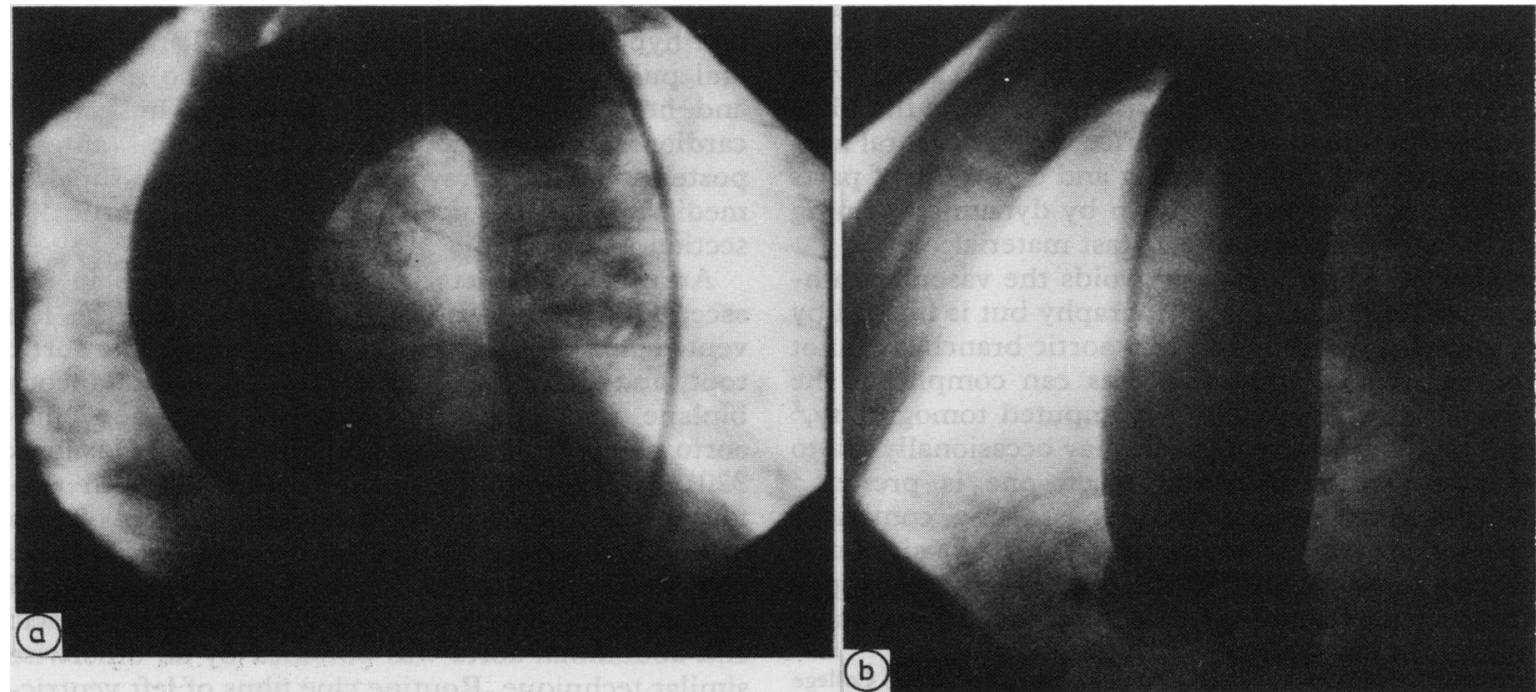

Fig 2 Aortograms in left anterior oblique (a) and lateral (b) projections in case 1. There are no features to indicate dissection. 

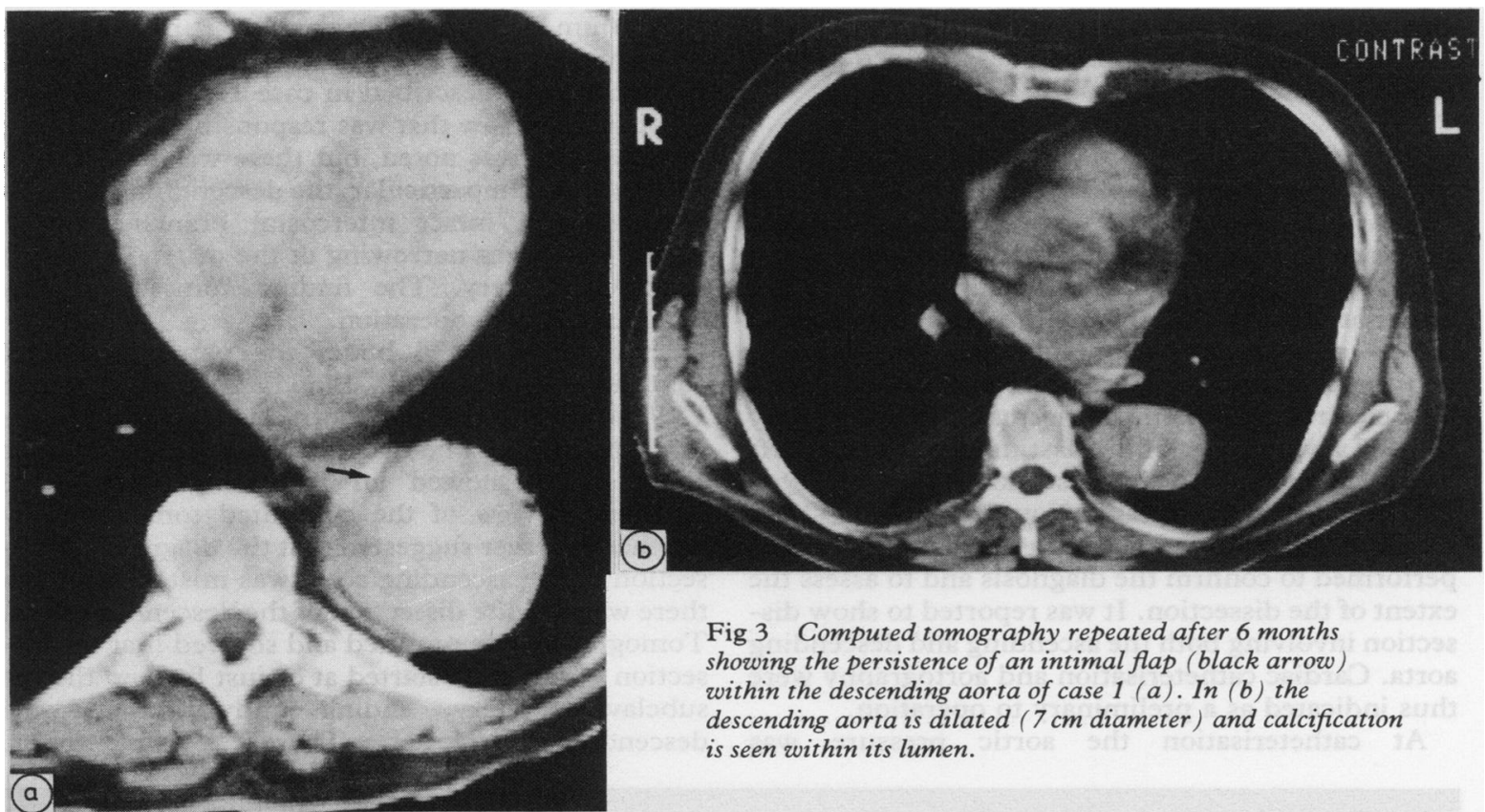

Fig 3 Computed tomography repeated after 6 months showing the persistence of an intimal flap (black arrow) within the descending aorta of case $1(a)$. In (b) the descending aorta is dilated ( $7 \mathrm{~cm}$ diameter) and calcification is seen within its lumen.

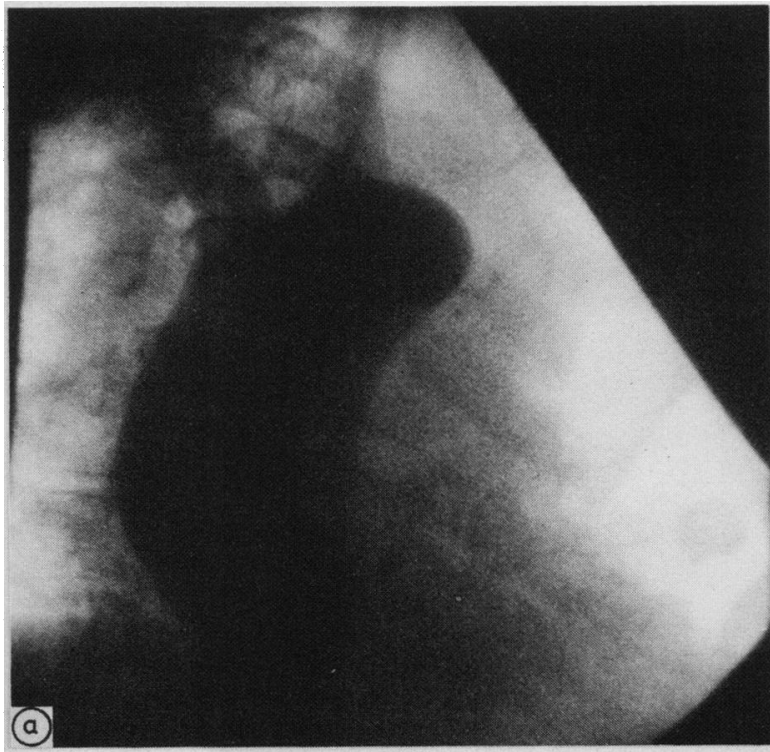

Fig 4 Aortograms showing the ascending aorta and aortic arch ( $a$, right anterior oblique projection) and the descending thoracic aorta ( $b$, posteroanterior projection) in case 2. The extra-aortic soft tissue shadow from mediastinal haematoma is visible (a). There is no direct evidence of dissection.

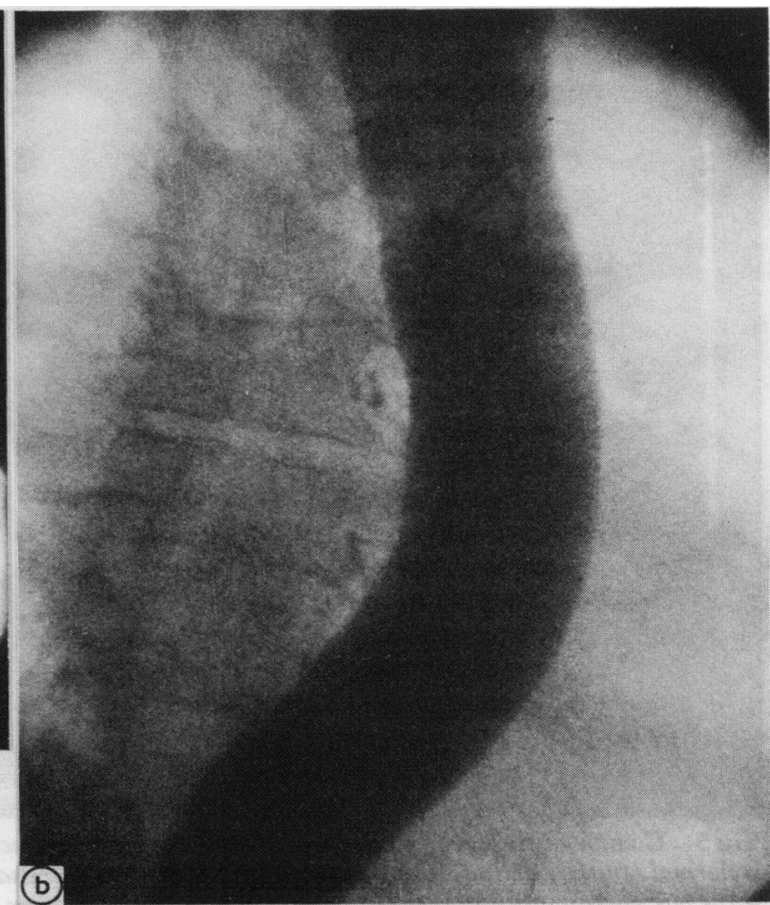


sign of dissection was apparent.

The patient remains well and has declined further investigation.

\section{CASE 2}

Sudden severe interscapular pain developed in a 72 year old man while he was gardening. The pain persisted and he was admitted to hospital the next day. Hypertension had been noted in the past but was not treated. His blood pressure was normal $(120 / 80 \mathrm{~mm} \mathrm{Hg})$, all peripheral pulses were present, and there was no cardiac failure and no murmurs. The electrocardiogram was normal. The chest $x$ ray showed a wide superior mediastinum and a small left pleural effusion. Aortic dissection (probably type III, DeBakey's classification ${ }^{6}$ ) was diagnosed. Computed tomography with contrast enhancement was performed to confirm the diagnosis and to assess the extent of the dissection. It was reported to show dissection involving both the ascending and descending aorta. Cardiac catheterisation and aortography were thus indicated as a preliminary to operation.

At catheterisation the aortic pressure was
$130 / 80 \mathrm{~mm} \mathrm{Hg}$ and left ventricular pressure was $130 / 5 \mathrm{~mm} \mathrm{Hg}$. Three aortograms were obtained by the techniques described in case 1 . An extra-aortic soft tissue shadow that was responsible for the wide mediastinum was noted, but there was no evidence of dissection. In particular, the descending aorta was normal, with intact intercostal branches (fig 4). There was slight narrowing at the origin of the left subclavian artery. The findings on aortography deterred us from operation.

Over the next 36 hours the patient's clinical condition deteriorated. Blood pressure rose to $170 / 110 \mathrm{~mm} \mathrm{Hg}$ and was controlled by nitroprusside infusion. He became dyspnoeic and wheezy. Serial chest $x$ rays showed an increasing left pleural effusion. Review of the computed tomograms by another observer suggested that the diagnosis of dissection in the ascending aorta was mistaken though there was definite dissection of the descending aorta. Tomography was repeated and showed that the dissection of the aorta started at or just beyond the left subclavian artery, ending midway down the descending thoracic aorta. There was visible leakage

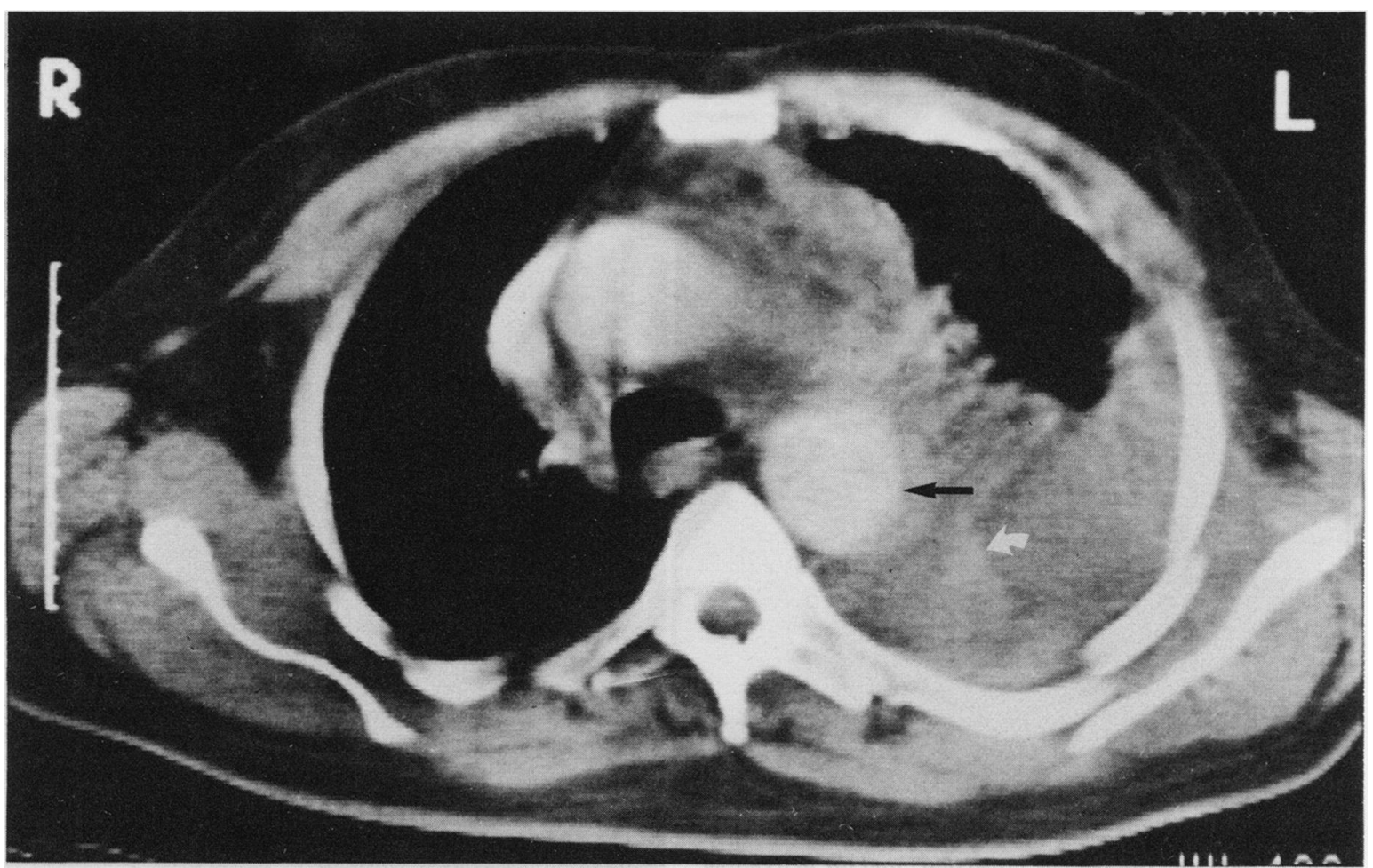

Fig 5 Computed tomography of thorax, after contrast enhancement, caudal to the aortic arch in case 2. The ascending aorta is normal. In the descending aorta contrast medium fills two channels separated by an intimal flap (black arrow). Within the large left pleural effusion there is an area of higher attenuation (curved arrow) showing contrast medium that has leaked from the aorta. 
of contrast medium from the aorta into the left hemithorax (fig 5). An operation was performed immediately.

At operation a large amount of fresh and clotted blood was found in the left chest, and an extensive mediastinal haematoma was confirmed. The dissecting aneurysm was identified; it extended from just distal to the left subclavian artery to $7-8 \mathrm{~cm}$ into the descending aorta. There was no evidence of dissection in the ascending aorta. The aorta was cross clamped above and below the dissection and a $26 \mathrm{~mm}$ woven Dacron graft was sutured into place. Six months after operation the patient is leading an active life.

\section{Discussion}

In case 1 the clinical details were suggestive of aortic dissection. As was then our practice, the initial investigation was by cardiac catheterisation and aortography. Although the study was of good quality, no evidence of dissection was found. Continuing clinical suspicion of dissection led us to perform computed tomography, and as a result the patient was referred to another institution for operation. There, normal findings on repeat aortography were interpreted as excluding the diagnosis of dissection and operation was not undertaken. Therefore we do not have surgical confirmation of the diagnosis of dissection, but the diagnosis is irrefutable on the follow up tomographic study (fig 3 ).

In case 2 the clinical details were suggestive of a type III dissection (DeBakey's classification). Computed tomography confirmed the diagnosis of dissection but was reported as showing dissection also in the ascending aorta (therefore type I). Because type I dissection is managed by operation, cardiac catheterisation was undertaken before surgery. Good quality aortograms, however, showed no evidence of dissection. Computed tomography was repeated because of deterioration in the patient's clinical condition; it showed a type III dissection leaking into the left hemithorax. On preoperative review, the first tomographic report of involvement of the ascending aorta was considered to have been mistaken. The findings at operation confirmed the findings of the second computed tomography.

In a shocked and deteriorating patient with severe aortic regurgitation or tamponade or both caused by proximal aortic dissection, cross sectional, echocardiography at the bedside may be all that is possi- ble before the patient is taken to theatre. Our experience with computed tomography leads us to regard it as the preferred initial diagnostic method in the more stable patient. If tomography shows that the dissection is confined to the descending aorta and there are no complications such as mediastinal compression and continuing haemorrhage into the thorax (as in case 2), medical management is appropriate and aortography may be avoided. If, however, dissection affects the ascending aorta, cardiac catheterisation to assess the severity of aortic regurgitation and to obtain high resolution images of the coronary arteries and aortic arch branches is generally regarded as a prerequisite of operation. Aortography is better than computed tomography in localising the intimal tear, but this may not be essential to operative management. ${ }^{7}$

Aortography is generally regarded as the definitive investigation for acute dissection of the aorta but false negative results have been reported. ${ }^{8}$ Computed tomography is a reliable alternative diagnostic method, and the two cases reported here suggest that it is in fact superior to aortography.

\section{References}

1 Godwin JD, Korobkin M. Acute disease of the aorta. Diagnosis by computed tomography and ultrasonography. Radiol Clin North Am 1983;21: 551-74.

2 Danza FM, Fusco A, Falappa P. The role of computed tomography in the evaluation of dissecting aortic aneurysms. Radiology 1984;152:827-9.

3 Thorsen MK, San Dretto MA, Lawson TL, Foley WD, Smith DF, Berland LL. Dissecting aortic aneurysms: accuracy of computed tomographic diagnosis. Radiology 1983;148:773-7.

4 Oudkerk M, Overbosch E, Dee P. CT recognition of acute aortic dissection. Am F Radiol 1983;141:671-6.

5 Neumann CH, Guthaner DF, Wong DWH. Aortic dissection: CT and angiographic comparisons. Digit Bilddiagn 1984;4:14-7.

6 DeBakey ME, McCollum CH, Crawford ES, et al. Dissection and dissecting aneurysms of the aorta: twenty-year follow-up of five hundred twenty-seven patients treated surgically. Surgery 1982;92:1118-34.

7 Miller DC, Stinson EB, Oyer PE, et al. Operative treatment of aortic dissection. Experience with 125 patients over a sixteen-year period. $\mathcal{f}$ Thorac Cardiovasc Surg 1979;78:365-82.

8 Shuford WH, Sybers RG, Weens HS. Problems in the aortographic diagnosis of dissecting aneurysm of the aorta. N Engl f Med 1969;280:225-31. 